\title{
J. F. Keggin, J. A. Santos et al. - No Octogésimo Aniversário da Determinação da Estrutura do Anião de KegGin
}

\author{
Ana M. V. Cavaleiro*, Helena I. S. Nogueira \\ CICECO, Universidade de Aveiro \\ anacavaleiro@ua.pt
}

Dedicado a M. T. Pope, no seu octogésimo aniversário

\begin{abstract}
A estrutura molecular do anião do ácido fosfotúngstico foi descrita pela primeira vez por J. F. Keggin numa carta à revista Nature datada de 10 de Maio de 1933. A resolução do problema da estrutura de alguns heteropoliácidos e seus sais resultou do trabalho desenvolvido por vários jovens investigadores na Universidade de Manchester entre os quais se encontrava um português. Neste contexto, após o enquadramento do anião de Keggin e outros relacionados no campo dos polioxometalatos, refere-se o trabalho realizado para resolver o problema da fórmula química e estrutura de heteropoliácidos e relembra-se a participação portuguesa neste estudo pioneiro, passados 80 anos sobre a data da primeira publicação.
\end{abstract}

\section{INTRODUÇÃO AOS POLIOXOMETALATOS}

A formação de polioxoaniões é um aspecto importante e de grande interesse da química do molibdénio e tungsténio em estados de oxidação elevados. Em soluções aquosas alcalinas de $\mathrm{Mo}(\mathrm{VI})$ ou $\mathrm{W}(\mathrm{VI})$ predominam os oxoaniões molibdato $\left(\mathrm{MoO}_{4}^{2-}\right)$ e tungstato $\left(\mathrm{WO}_{4}^{2-}\right)$, respectivamente, mas o abaixamento de $\mathrm{pH}$ provoca reaç̧ões de condensação com formação de diversos oxoiões polinucleares, dependendo da concentração, pH e tipo de ácido adicionado (Figura 1). Em soluções aquosas ácidas dificilmente se encontram espécies mononucleares na ausência de agentes complexantes. $\mathrm{O}$ processo de polimerização está associado à tendência dos aniões $\mathrm{MO}_{4}{ }^{2-}$ protonados $(\mathrm{M}=\mathrm{Mo}, \mathrm{W})$ expandirem a sua esfera de coordenação de 4 para 6 (ou 5) e das unidades $\mathrm{MO}_{\mathrm{x}}$ resultantes se agregarem por partilha de átomos de oxigénio (Figura 2A). Se as espécies poliméricas forem do tipo $\left[\mathrm{H}_{\mathrm{p}} \mathrm{M}_{\mathrm{q}} \mathrm{O}_{\mathrm{x}}\right]^{\mathrm{n}-}, \mathrm{M}=\mathrm{Mo}, \mathrm{W}$, são designadas por isopolianiões. Quando em solução existem espécies aniónicas ou catiónicas susceptíveis de se ligarem por pontes de oxigénio ao metal $M$ formam-se espécies referidas por heteropolianiões, de fórmula $\left[\mathrm{H}_{\mathrm{p}} \mathrm{Z}_{\mathrm{z}} \mathrm{M}_{\mathrm{q}} \mathrm{O}_{\mathrm{x}}\right]^{\mathrm{n}}$, onde $\mathrm{Z}$ é um elemento diferente de $\mathrm{M}$, que pode ser não metálico ou semimetálico, como P, Si ou B, ou um metal como Al, Co ou Fe. Estes tipos de aniões são hoje incluídos no largo conjunto de espécies que se designam por polioxometalatos.

No seu global, os polioxometalatos (POMs) são agregados de oxigénio e metais $M$ do grupo 5 e 6 (com excepção do crómio), que podem incorporar outros elementos de quase todos os grupos da tabela periódica. Os polioxometalatos apresentam estrutura e composição muito variadas (Figura 2B) e são frequentemente designados por nomes de investigadores que participaram no seu estudo. Os elementos $M$ (em estados de oxidação de +4 a +6 ) encontram-se rodeados de iões óxido, em grupos $\mathrm{MO}_{\mathrm{x}}$ que partilham entre si vértices e arestas (raramente faces). Os iões metálicos não se encontram no centro dos poliedros $\mathrm{MO}_{\mathrm{x}}$, mas sim deslocados para um vértice ou aresta devido à ocorrência de ligações $\pi$ metal-oxigénio. O número de ligações deste tipo, por átomo M, é normalmente 1 ou 2. Os restantes elementos estão igualmente ligados a oxigénio no centro de poliedros de coordenação (tetraedros, octaedros, etc.). A combinação de polioxometalatos com catiões orgânicos ou inorgânicos e um sem número de outras moléculas conduz a uma multiplicidade de compostos de propriedades e aplicações muito diversas. Podemos usar a palavra polioxometalatos para designar quer os aniões quer os compostos contendo estes aniões. M. T. Pope, no seu inultrapassável livro "Heteropoly and Isopoly Oxometalates" apresenta-nos o que era conhecido até ao início da década de 1980 [1], após o que os POMs têm sido referidos em vários livros [2-4]. Um abrangente fascículo temático do Chemical Reviews foi publicado em 1998 [5]. O estado actual do conhecimento foi tema de diversos artigos de revisão publicados na Chemical Society Reviews em 2012 [6].
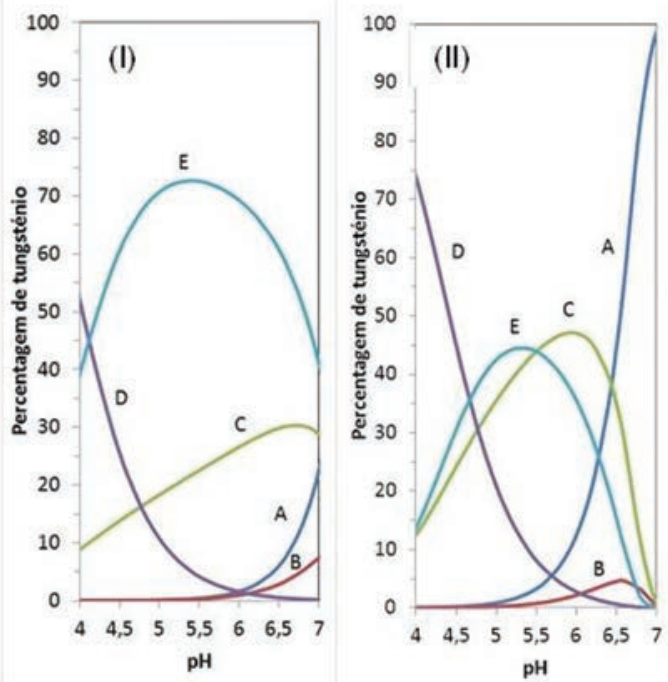

Figura 1 - Concentração de tungsténio(VI) nas diversas espécies, em função do $\mathrm{pH}$, expressa em percentagem da concentração total em tungsténio: (I) $0,1 \mathrm{~mol} \mathrm{dm}^{-3}$; (II) $0,01 \mathrm{~mol} \mathrm{dm}^{-3}$. A: $\mathrm{WO}_{4}^{2-;}$ B: $\left[\mathrm{W}_{6} \mathrm{O}_{20}(\mathrm{OH})_{2}\right]^{6--}$; C: $\left[\mathrm{W}_{7} \mathrm{O}_{24}\right]^{6-} ; \mathrm{D}:\left[\mathrm{HW}_{7} \mathrm{O}_{24}\right]^{6-} ; \mathrm{E}:\left[\mathrm{H}_{2} \mathrm{~W}_{12} \mathrm{O}_{42}\right]^{10-}$. Diagramas calculados com base nas constantes de equilíbrio da seguinte referência: J. J. Cruywagen, I. F. J. Merwe, J. Chem. Soc., Dalton Trans. (1987) 1701-1705 
(A)

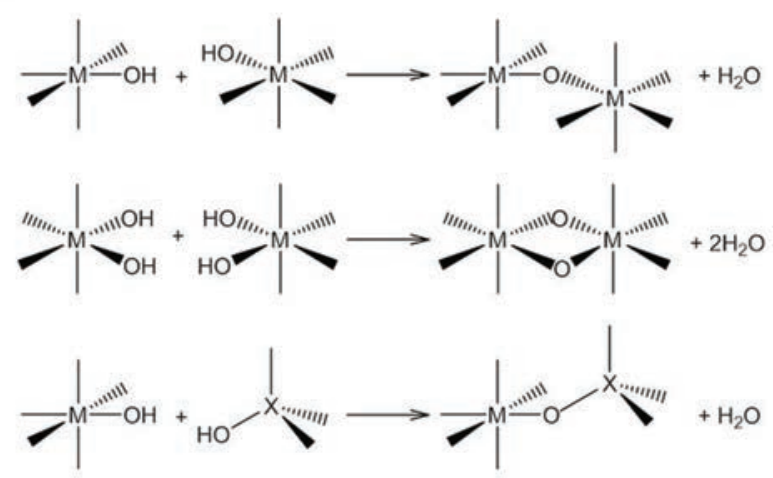

(B)

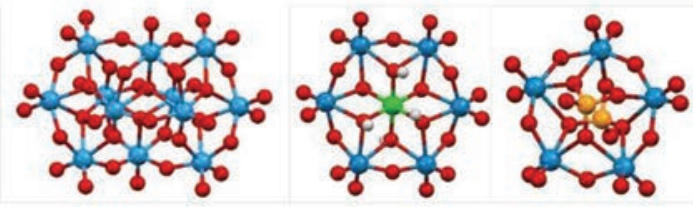

Figura 2 - (A) Exemplos de reacções de condensação conducentes à formação de espécies polinucleares. (B) Estrutura molecular de alguns POMs. Da esquerda para a direita: paratungstato $\mathrm{B},\left[\mathrm{H}_{2} \mathrm{~W}_{12} \mathrm{O}_{42}\right]^{10-} ; \mathrm{POM}$ do tipo Anderson $\left[\mathrm{CrMo}_{6} \mathrm{O}_{24} \mathrm{H}_{6}\right]^{3-} ; \mathrm{POM}$ do tipo Strandberg, $\left[\mathrm{Mo}_{5} \mathrm{P}_{2} \mathrm{O}_{23}\right]^{6-}$ Azul: metal M = Mo ou W; vermelho: O; laranja: P; verde: $\mathrm{Cr}$; branco: $\mathrm{H}$.

\section{O anIÃO de KegGin e SeUS deRIVAdos}

Os polioxometalatos do tipo Keggin formam um grupo interessante de heteropolianiões com aplicações em campos tão diversos como catálise, medicina e ciência de materiais [7-12]. Na base desta classe encontram-se os polioxometalatos de fórmula $\left[\mathrm{XM}_{12} \mathrm{O}_{40}\right]^{\mathrm{n}-},(\mathrm{M}=\mathrm{Mo} \mathrm{e} \mathrm{W}, \mathrm{X}=\mathrm{P}, \mathrm{As}, \mathrm{B}, \mathrm{Si}$, $\mathrm{Ge}, \mathrm{Al}, \mathrm{Fe}, \mathrm{Co}$, etc.), que hoje são designados por aniões de Keggin. Nela se inclui o primeiro polioxometalato de que há notícia, o anião $\left[\mathrm{PMo}_{12} \mathrm{O}_{40}\right]^{3-}$, preparado na forma de sal de amónio $\left(\mathrm{NH}_{4}\right)_{3}\left[\mathrm{PMo}_{12} \mathrm{O}_{40}\right] \cdot \mathrm{mH}_{2} \mathrm{O}$ no primeiro quartel do século XIX [1]. A estrutura dos aniões de Keggin (Figura 3) foi desvendada na década de 1930 mediante estudos de difracção de raios $\mathrm{X}$ de pós (vide infra). Como subsequentemente se identificaram isómeros para os aniões $\left[\mathrm{XM}_{12} \mathrm{O}_{40}\right]^{\mathrm{n}-}$, a estrutura representada na Figura 3 designa-se por $\alpha$-Keggin [1]. A Figura 3C evidencia o empacotamento compacto de iões óxido, já que os polioxometalatos são muitas vezes considerados como análogos moleculares de redes de óxidos metálicos. Na continuação deste texto vamos omitir o prefixo $\alpha$ porque apenas vamos referir este isómero.

Para além dos polioxometalatos $\left[\mathrm{XM}_{12} \mathrm{O}_{40}\right]^{\mathrm{n}-}$, os aniões do tipo Keggin incluem derivados lacunares e muitas outras es-

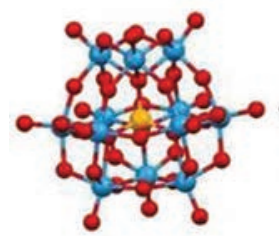

(A)

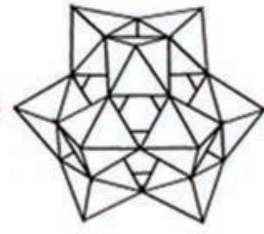

(B)

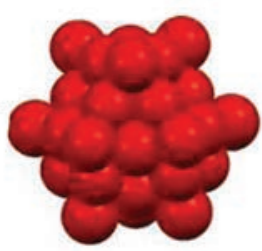

(C)
Figura 3 - Várias representações da estrutura do anião $\alpha$-Keggin pécies estruturalmente relacionadas [1]. Um derivado lacunar mantém o motivo estrutural dos aniões de Keggin, mas apresenta lacunas resultantes da ausência de alguns átomos M e correspondentes átomos de oxigénio terminal e outros (Figura 4). Os derivados lacunares podem-se ligar a iões metálicos, formando complexos com estruturas variadas, como exemplificado na Figura 4. Os aniões monolacunares $\left[\mathrm{XM}_{11} \mathrm{O}_{39}\right]^{\mathrm{n}-}$ ligam-se a um metal por 4 ou 5 átomos de oxigénio, formando, por exemplo, derivados monosubstituídos $\left[\mathrm{XM}_{11} \mathrm{M}^{\prime}\left(\mathrm{H}_{2} \mathrm{O}\right) \mathrm{O}_{39}\right]^{\mathrm{n}-}\left(\mathrm{X}=\mathrm{P}, \mathrm{Si}\right.$, As, etc, $\mathrm{M}^{\prime}=$ metal de transição ou do bloco $p$ ), onde $\mathrm{M}^{\prime}$ ' se encontra ainda coordenado a uma molécula de $\mathrm{H}_{2} \mathrm{O}$, ou $\left[\mathrm{M}^{\prime}\left(\mathrm{XM}_{11} \mathrm{O}_{39}\right)_{2}\right]^{\mathrm{n}-}$, com M' = lantanídeo ou actinídeo. Este tipo de aniões começou a ser estudado nos finais da década de 1950 [13], mais de 100 anos depois da preparação dos primeiros POMs. Os aniões trilacunares $\left[\mathrm{XM}_{9} \mathrm{O}_{34}\right]^{\mathrm{n}}$, por sua vez, apresentam 6 ou 7 átomos de oxigénio potencialmente coordenantes (dependendo do isómero considerado, A ou B), ligando-se a agrupamentos polinucleares de metais, como, por exemplo, nos aniões sanduíche $\left[\mathrm{M}_{4}\left(\mathrm{H}_{2} \mathrm{O}\right)_{2}\left(\mathrm{PW}_{9} \mathrm{O}_{34}\right)_{2}\right]^{10-}, \mathrm{M}=\mathrm{Mn}$, $\mathrm{Co}, \mathrm{Cu}, \mathrm{Zn}$, etc, e outros [1].

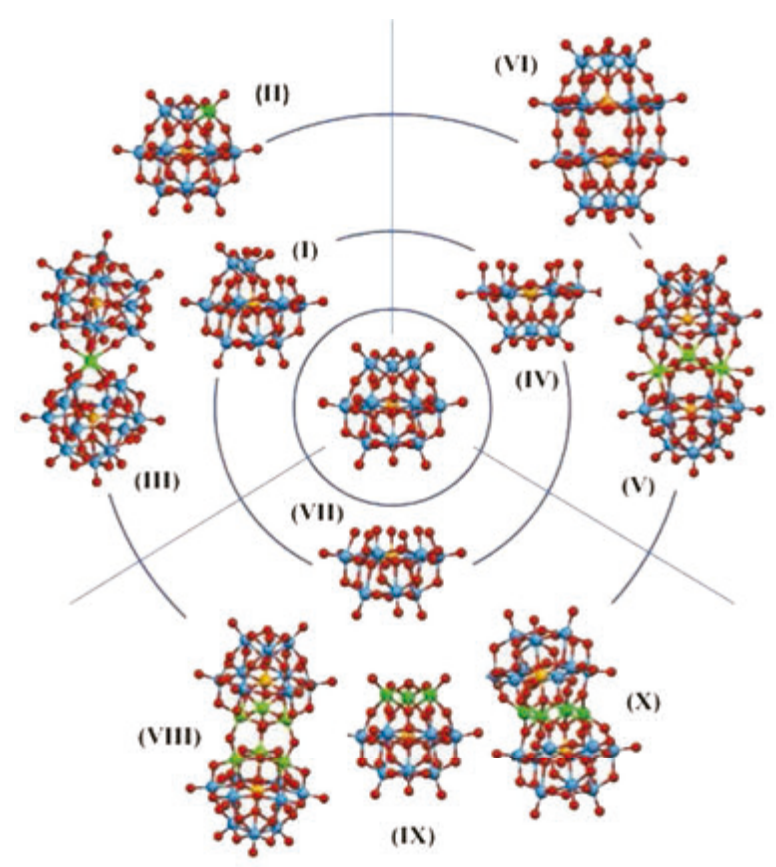

Figura 4 - Estrutura de aniões do tipo Keggin. O anião $\alpha$-Keggin está no centro. Os aniões lacunares dispõem-se no círculo interior. $\mathrm{O}$ anião de Wells-Dawson e diversos complexos metálicos encontram-se sobre o círculo exterior. Terço superior esquerdo: (I) $\left[\mathrm{XM}_{11} \mathrm{O}_{39}\right]^{\mathrm{n}-}$; (II)

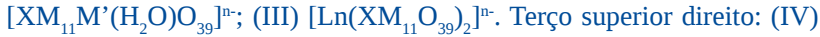
A- $\left[\mathrm{XM}_{9} \mathrm{O}_{34}\right]^{\mathrm{n}-} ;(\mathrm{V})\left[\mathrm{M}_{3}\left(\mathrm{H}_{2} \mathrm{O}\right)_{3}\left(\mathrm{XW}_{9} \mathrm{O}_{34}\right)_{2}\right]^{\mathrm{n}-} ;(\mathrm{VI})\left[\mathrm{X}_{2} \mathrm{M}_{18} \mathrm{O}_{68}\right]^{\mathrm{n}-}$. Terço inferior: (VII) B-[ $\left[\mathrm{XM}_{9} \mathrm{O}_{34}\right]^{\mathrm{n}-}$; (VIII) $\left[\left(\mathrm{SiW}_{9} \mathrm{O}_{34} \mathrm{Fe}_{3}(\mathrm{OH})_{3}\right)_{2}(\mathrm{OH})_{3}\right]^{11-} ;$ (IX) $\left[\mathrm{XM}_{9} \mathrm{M}_{3}{ }_{3}\left(\mathrm{H}_{2} \mathrm{O}\right)_{3} \mathrm{O}_{37}\right]^{\mathrm{n}-}$; (X) $\left[\mathrm{M}_{4}\left(\mathrm{H}_{2} \mathrm{O}\right)_{2}\left(\mathrm{XW}_{9} \mathrm{O}_{34}\right)_{2}\right]^{\mathrm{n}-} \cdot \mathrm{M}=\mathrm{Mo}$ ou W, M' $=$ metal de transição ou do bloco $p, \mathrm{Ln}=$ lantanídeo. Azul: metal M; vermelho: O; laranja: X; verde: $M^{\prime}$

É curioso verificar que existem muitas outras espécies que derivam ou contêm fragmentos de aniões de Keggin, que parecem combinar-se de um sem número de modos, quais peças de Lego molecular. Por exemplo, os chamados aniões de Wells-Dawson, [ $\mathrm{X}_{2} \mathrm{M}_{18} \mathrm{O}_{68}{ }^{\mathrm{n}}{ }^{\text {- }},(\mathrm{X}=\mathrm{P}, \mathrm{As}, \mathrm{S})$ [1], parecem resultar da fusão de dois aniões de Keggin trilacunares (Figura 4). De modo análogo ao que ocorre com os aniões de 
Keggin, a família de aniões de Wells-Dawson inclui derivados lacunares e seus complexos metálicos [14].

O estudo dos compostos com catiões variados e aniões do tipo Keggin ou relacionados disparou nas últimas décadas, conhecendo-se agora uma multiplicidade de POMs e de compostos com estruturas cristalinas muito diversificadas [15-17]. De esta multiplicidade de composição e estrutura resultam propriedades muito diversificadas potenciando a possibilidade de usar estes compostos em aplicações muito variadas. O nosso interesse pelos aniões do tipo Keggin está na origem do trabalho de investigação que temos vindo a desenvolver em Aveiro [18-20].

\section{OS HETEROPOLIÁCIDOS}

Os compostos com aniões de Keggin de fórmula $\mathrm{H}_{\mathrm{n}}\left[\mathrm{XM}_{12} \mathrm{O}_{40}\right] \cdot \mathrm{mH}_{2} \mathrm{O}(\mathrm{X}=\mathrm{P}, \mathrm{Si}, \mathrm{As}, \mathrm{Ge}$, etc) incluem-se no que se costuma designar por heteropoliácidos (que também abrangem os ácidos de Wells-Dawson $\left.\mathrm{H}_{\mathrm{n}}\left[\mathrm{X}_{2} \mathrm{M}_{18} \mathrm{O}_{68}\right] \cdot \mathrm{mH}_{2} \mathrm{O}\right)$. Foi o estudo da estrutura cristalina de heteropoliácidos, particularmente do ácido fosfotúngstico (ou dodecatungstofosfórico), que conduziu à determinação da estrutura do anião $\alpha$-Keggin [21, 22]. Os heteropoliácidos $\mathrm{H}_{\mathrm{n}}\left[\mathrm{XM}_{12} \mathrm{O}_{40}\right] \cdot \mathrm{mH}_{2} \mathrm{O}$ cristalizam com elevado e variável número de moléculas de água de hidratação, dependendo das condições experimentais, nomeadamente da temperatura. A cristalização à temperatura ambiente pode originar compostos cúbicos com $\mathrm{m}=29$-30 (que eflorescem com perda de cristalinidade), ou triclínicos, mais estáveis, com 13-14 $\mathrm{H}_{2} \mathrm{O}$ [23]. O aquecimento (ou outro método adequado) conduz à forma hexa-hidratada, cúbica [23, 24].

As fórmulas químicas vulgarmente usadas para estes ácidos (por exemplo, $\mathrm{H}_{3} \mathrm{PW}_{12} \mathrm{O}_{40} \cdot \mathrm{xH}_{2} \mathrm{O}$ ou $\mathrm{H}_{3} \mathrm{PW}_{12} \mathrm{O}_{40} \cdot a q$ ) mascaram completamente o facto de que nos compostos sólidos os heteropolianiões se encontram geralmente desprotonados, organizados numa rede em que os contra-catiões são protões hidratados como, por exemplo, $\left[\mathrm{H}_{5} \mathrm{O}_{2}\right]^{+}[1]$. Este arranjo confere-lhes propriedades ácidas no estado sólido e, em certos casos, elevada condutividade eléctrica [24]. Os heteropoliácidos de Keggin são ácidos fortes, com extensas aplicações em catálise ácida [25].

No contexto da estrutura dos heteropoliácidos e seus sais, a estrutura do anião é designada por estrutura primária. Estrutura secundária designa o arranjo observado nos compostos sólidos de aniões, catiões e outras moléculas presentes, como, por exemplo, água de hidratação [26]. No campo da catálise heterogénea considera-se ainda a estrutura terciária, que é definida por características do sólido como tamanho de partícula, porosidade e área superficial [27].

\section{MAS QUEM É KEgGIn?}

O trabalho realizado por Keggin que conduziu ao conhecimento da estrutura do anião do ácido fosfotúngstico foi efectuado em Manchester, no laboratório de Física da Uni- versidade [28]. À época, sob a direcção de W. L. Bragg, o laboratório em Manchester era um importante centro de estudo de estruturas inorgânicas, nomeadamente de silicatos e ligas metálicas [29, 30]. É hoje bem conhecido o papel dos Bragg, pai e filho, no desenvolvimento da cristalografia de raios X e do estudo de estruturas cristalinas. Sir William Lawrence Bragg (o filho), por ocasião do seu octogésimo aniversário, relembrou os seus anos na Universidade de Manchester (1919 a 1937), revendo os desenvolvimentos técnicos e teóricos, assim como os resultados mais relevantes, que surgiram nesse período [30]. Não faltou nessa revisão, intitulada “Manchester days”, uma alusão à determinação da estrutura do anião do ácido fosfotúngstico efectuada por J. F. Keggin e à elevada simetria do anião [30]. "Manchester days" inclui a lista dos investigadores que passaram pelo laboratório de Física, o que nos permite saber que J. F. Keggin entrou para o grupo no ano lectivo de 1931-1932, no mesmo ano de alguém chamado J. R. de A. Santos [30].

Podemos seguir o percurso de Keggin em Manchester através das suas publicações e simultaneamente observar o desenrolar do trabalho feito no laboratório de Física para resolver o problema da estrutura dos heteropoliácidos e seus sais. Em 1933 Keggin publica duas cartas na revista Nature, sobre a estrutura cristalina de duas formas cúbicas do ácido fosfotúngstico, com 6-7 e 30 moléculas de água de hidratação, respectivamente [21, 31]. Na primeira destas cartas, datada de 10 de Maio (Figura 5), Keggin descreve sucintamente e pela primeira vez, a estrutura do anião do ácido fosfotúngstico [21]. Nela se pode ler o seguinte:

"The structure of 12-phosphotungstic acid has recently been found in this laboratory by X-ray analysis, using the powder method. The phosphorus atom is at the centre of a regular tetrahedral group of four oxygen atoms, and each tungsten atom is approximately at the centre of a distorted octahedral group of six oxygen atoms. (...) The complex anion is found experimentally to be $\mathrm{PW}_{12} \mathrm{O}_{40}{ }^{-3}$ and has the point group symmetry $T_{d}$. The twelve $\mathrm{WO}_{6}$ octahedra are arranged in four groups. (...) Four such groups of three octahedra are arranged round the central $\mathrm{PO}_{4}$ tetrahedron in tetrahedral symmetry, so that each oxygen of the $\mathrm{PO}_{4}$ tetrahedron is now an oxygen already shared between three $\mathrm{WO}_{6}$ octahedra."

A descrição mais completa foi publicada em 1934 [22]. Keggin atribuiu ao hidrato estudado a fórmula $\mathrm{H}_{3} \mathrm{PW}_{12} \mathrm{O}_{40} \cdot 5 \mathrm{H}_{2} \mathrm{O}$. Convém notar que, à época, as fórmulas químicas dos heteropoliácidos não eram ainda conhecidas com exactidão. Só em 1977 ficou esclarecido qual a verdadeira fórmula molecular deste ácido fosfotúngstico, no artigo de A. Levy e colaboradores intitulado "Dodecatungstophosphoric Acid Hexahydrate, $\left(\mathrm{H}_{5} \mathrm{O}_{2}{ }^{+}\right)_{3}\left(\mathrm{PW}_{12} \mathrm{O}_{40}{ }^{3-}\right)$. The True Structure of Keggin's "Pentahydrate”, from Single-Crystal X-ray and Neutron Diffraction Data” [32]. Apesar dos métodos mais rigorosos não confirmarem as dimensões interatómicas determinadas por Keggin, a estrutura do anião (Figura 6) perdura até hoje. 

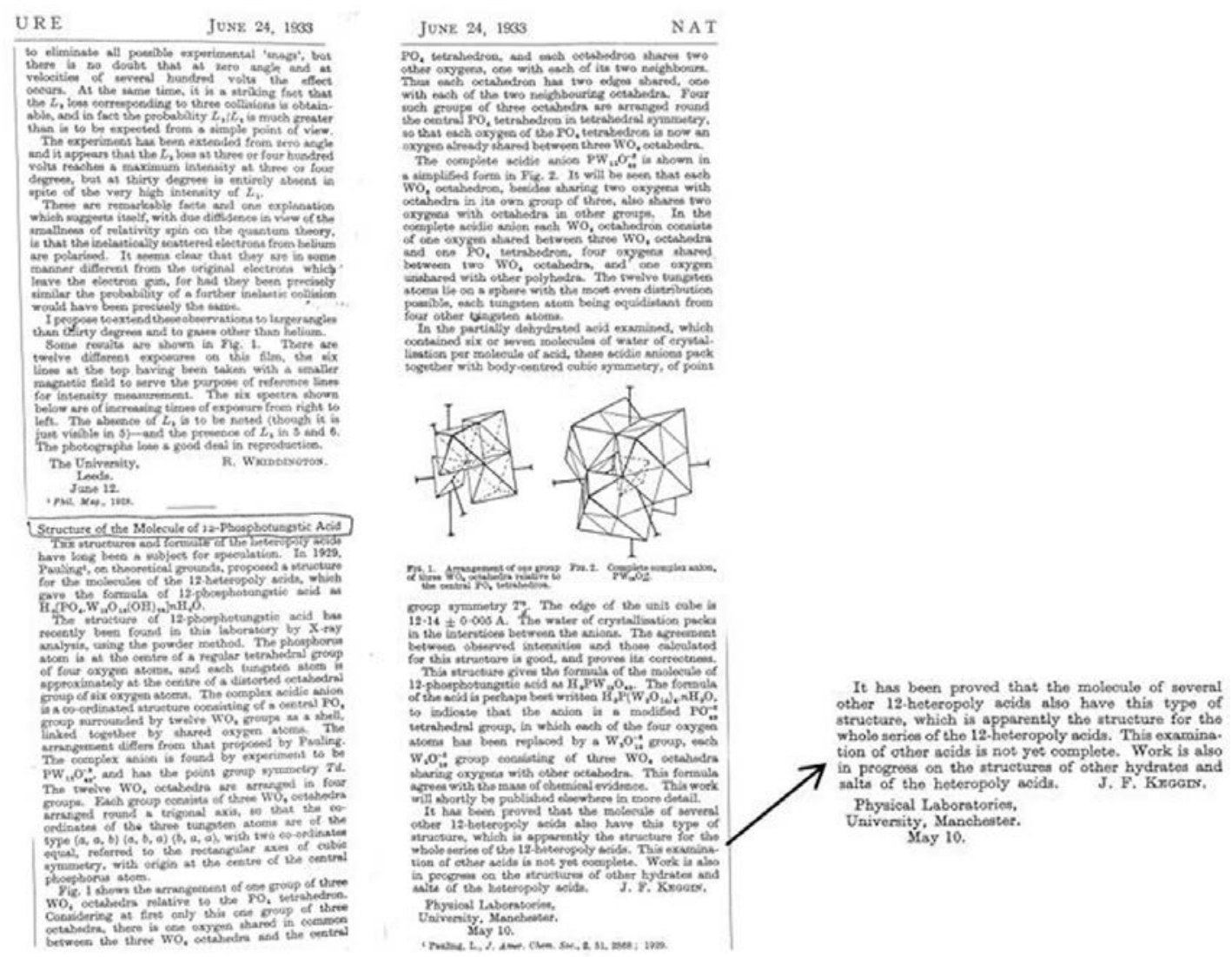

Figura 5 - Páginas da revista Nature com a comunicação de J. F. Keggin (Nature, 131 (1933) 908-909)

(A)

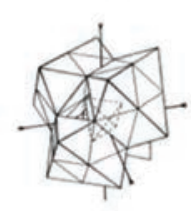

(C)

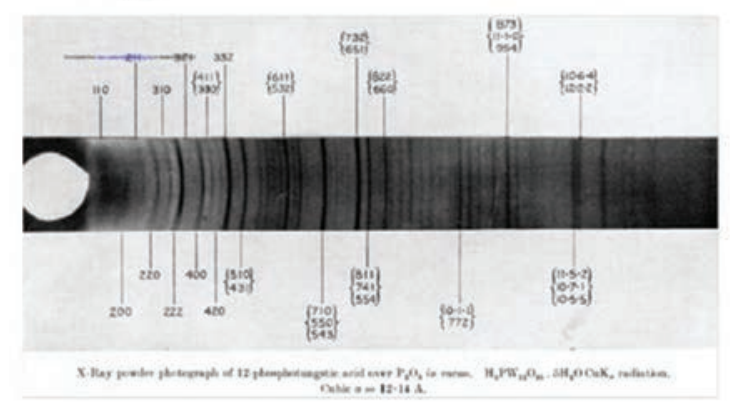

(D)

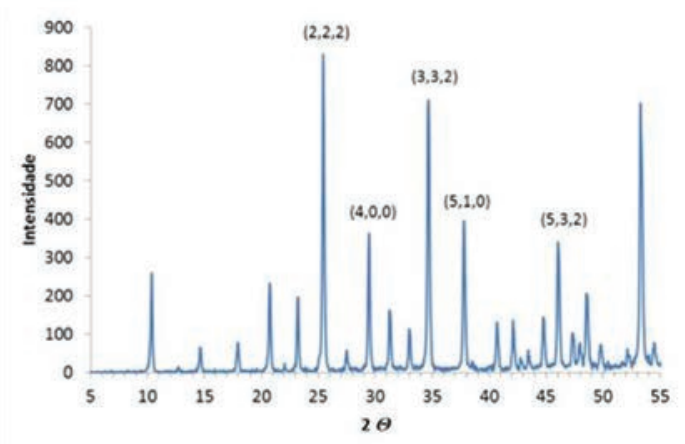

Um último artigo sobre heteropoliácidos, da autoria de J. W. Illingworth e Keggin, surge em 1935 [33]. Intitulado "Identification of the 12-heteropoly-acids and their salts by means of X-Ray Powder Photographs”, o artigo começa por referir o trabalho de base já realizado naquele laboratório, ou seja, os artigos de Keggin [33], Signer e Gross [34] e Santos [35], após o que lista um conjunto de heteropoliácidos e seus sais que cristalizam com a estrutura do ácido fosfotúngstico hexa-hidratado, identificados a partir do padrão de difracção de raios $\mathrm{X}$ de pós. Esta lista envolve espécies com os aniões $\left[\mathrm{XW}_{12} \mathrm{O}_{40}\right]^{\mathrm{n}-}, \mathrm{X}=\mathrm{P}, \mathrm{As}, \mathrm{Si}, \mathrm{Ge}, \mathrm{B}$, $2 \mathrm{H}$ e $\left[\mathrm{XMo}_{12} \mathrm{O}_{40}\right]^{\mathrm{n}-}, \mathrm{X}=\mathrm{P}, \mathrm{As}, \mathrm{Si}, \mathrm{Mn}(\mathrm{IV}), \mathrm{Ti}(\mathrm{IV})$, incluindo alguns ácidos, sais de amónio, de catiões de metais alcalinos e de $\mathrm{Tl}(\mathrm{I})$, num total de mais de trinta compostos.

A existência de várias formas hidratadas com estruturas diferentes e a dificuldade em determinar o número de moléculas de água de hidratação constituiu um problema para os investigadores de Manchester. Apesar da comunicação de Keggin em 1933 sobre o ácido fosfotúngstico com 29-30 moléculas de água [31], só em 1936 Bradley e Illingworth divulgaram a estrutura deste hidrato, com um agradecimento ao Dr. J. F. Keggin por ter fornecido uma fotografia da difracção de raios X de pó do composto [36]. Keggin tinha, correctamente, identificado que o anião nos

Figura 6 - Estrutura de Keggin do ácido fosfotúngstico, dito penta-hidratado: (A) Estrutura do anião $\left[\mathrm{PW}_{12} \mathrm{O}_{40}\right]^{3-}$; (B) arranjo cúbico de corpo centrado de aniões; (C) padrão de difração de raios X. Reproduzido de Proc. R. Soc. Lond. A, 144 (1934) 75-100, com autorização da Royal Society of Chemistry. Para comparação mostra-se em (D) o padrão de DRX de pós do $\mathrm{H}_{3}\left[\mathrm{PW}_{12} \mathrm{O}_{40}\right] \cdot 6 \mathrm{H}_{2} \mathrm{O}$ 
dois hidratos era o mesmo. No entanto o empacotamento de aniões é diferente nos dois hidratos, sendo o composto hexa-hidratado mais denso quando comparado com o mais hidratado (5,6 $\mathrm{g} \mathrm{cm}^{-3}$ versus $\left.3,6 \mathrm{~g} \mathrm{~cm}^{-3}\right)$.

Após esta data, o nome de J. F. Keggin não aparece mais como autor ligado ao meio académico. Foi possível ainda encontrá-lo nalguns artigos [37, 38] e na literatura de patentes, até cerca de 1955 . Nestes casos, as filiações apresentadas sugerem que Keggin desenvolveu a sua vida profissional na indústria.

É interessante notar que Keggin, um estudante de doutoramento [39], aparece como único autor na maioria das suas publicações de Manchester [21, 22, 31]. Bragg, o director do laboratório, e seu orientador (assim como de Santos) [39, 40], surge na frase “communicated by W. L. Bragg” no artigo publicado em Proceedings [22] e nos agradecimentos. A. J. Bradley, investigador com um papel preponderante na determinação de estruturas pelo método difracção de raios $X$ de pós [29, 30], também aparece apenas nos agradecimentos [22, 33].

A determinação da estrutura do anião $\left[\mathrm{PW}_{12} \mathrm{O}_{40}\right]^{3-}[21,22]$ e o reconhecimento de que aquela estrutura se mantém noutros heteropoliácidos e seus sais [33] constitui a importante contribuição de Keggin para a química estrutural dos polioxometalatos. Sabe-se que desde meados do século XIX se conheciam compostos com estes aniões e que diversas propostas de estrutura existiam à época do estudo de Keggin, que no seu artigo de 1934 faz uma revisão bibliográfica do que era conhecido. No seu conjunto, as referências [21] e [22] ultrapassam as mil citações, apesar de as designações “anião de Keggin” e "estrutura de Keggin” já terem encontrado o seu caminho para os livros de texto e serem apresentadas frequentemente sem referência ao trabalho original.

É de todo notável que o trabalho de Keggin tenha sido em grande parte confirmado nos estudos mais sofisticados realizado quarenta anos depois [32], e nas inúmeras determinações realizadas desde então [1]. É com toda a justiça que o seu nome é hoje recordado associado à estrutura que determinou. A estrutura secundária dos heteropoliácidos $\mathrm{H}_{\mathrm{n}}\left[\mathrm{XW}_{12} \mathrm{O}_{40}\right] \cdot \mathrm{mH}_{2} \mathrm{O}$, associada à variabilidade do grau de hidratação observado, continua a ser tema de estudo até aos dias de hoje [24].

Não deixa de ser curioso que não haja hoje menção de Bragg no campo dos polioxometalatos, apesar de pelo menos a determinação da estrutura primária dos aniões de Keggin e de Dawson (esta última no Cavendish Laboratory em Cambridge [41]) terem sido obtidas em laboratórios sob a sua direcção.

\section{E O tAL J. A. SANTOS?}

Ao contrário do que o título deste artigo parece sugerir, o nome de Keggin não aparece ligado ao de Santos na autoria de artigos científicos. Sabe-se que J. A. Santos trabalhou em Manchester, na mesma época de Keggin, no estudo de estrutura de silicatos (mencionado em [30]), do cloreto de cobalto(II) anidro [42] e dos sais de césio de diversos heteropoliácidos [35]. Deste trabalho surgiu ainda a proposta de uso do ácido fosfomolíbdico para a determinação gravimétrica de césio e potássio [43] e um artigo sobre difracção de raios X de pós a baixa temperatura [44].

No seu artigo sobre os sais de césio [35], Santos concluiu que todos os sais estudados tinham 3 catiões césio e apresentavam a estrutura cristalina do ácido fosfotúngstico, ou seja, que tinha preparado sais com a fórmula geral $\mathrm{Cs}_{3} \mathrm{H}_{\mathrm{n}-3} \mathrm{XW}_{12} \mathrm{O}_{40}$, onde n é a carga do anião e $\mathrm{X}=\mathrm{Si}, \mathrm{B}, 2 \mathrm{H}$. Nas suas palavras:

"An investigation by X-rays into the structure of caesium salts of several heteropolyacids has been carried out. The investigation follows upon earlier work by Keggin on the same structure of the 5-hydrate of phosphotungstic acid published in the "Proceedings of the Royal Society". It has been found that in the caesium salts the arrangement of the anions is the same of the 5-hydrate of the acid. The caesium atoms occupy the positions previously occupied by 3 of the 5 molecules of water of crystallization. (...) It is interesting to find that the number of caesium atoms in the molecules of silico-, boro-, and metatungstate is also 3. (....) Corresponding salts with rubidium and thalium, in place of caesium, (...) have the same structure.”

Santos talvez tivesse ficado surpreendido se soubesse que ainda hoje se estudam os sais de césio do ácido fosfotúngstico, que podem ocorrer com diversas estequiometrias [45], dado o seu interesse e utilização em catálise ácida, e que o seu artigo ainda é ocasionalmente citado [46].

Não foi difícil identificar o investigador com nome português que trabalhou com W. L. Bragg em Manchester no campo da cristalografia de raios $\mathrm{X}$ no início da década de 1930 [40, 47-49]. Trata-se de João Rodrigues de Almeida Santos, nascido em Viseu em Março de 1906 e falecido em 1975. Professor da Universidade de Coimbra, foi Director do Departamento de Física a partir de 1948. Hoje, dá nome à Biblioteca do Departamento de Física da Universidade de Coimbra. Santos doutorou-se na Universidade de Manchester em Janeiro de 1935 com a tese intitulada "An X-ray investigation into the structure of anhydrous cobaltous chloride, $\mathrm{CoCl}_{2}$ - at room and very low temperatures - and caesium, rubidium and thallium salts of certain 12-heteropoly acids” [40, 47-49]. Licenciado em Ciências Físico-Químicas com elevada classificação, Santos possuía as habilitações adequadas à resolução de um problema que envolvia ambas as disciplinas. Após o seu doutoramento regressou à Universidade de Coimbra, onde ainda desenvolveu investigação em estruturas de sólidos, tendo adquirido um aparelho para esse fim $[47,48]$. Aquando da prestação de provas para Professor Extraordinário, em 1947, apresentou uma dissertação sobre "Estudo, pelos raios X, do fosfotungstato de prata” [50]. Publicado em português, este trabalho não aparece mencionado nos artigos posteriores sobre o assunto [51]. O estudo do fosfotungstato 
de prata ocupou-o durante mais algum tempo, mas os seus interesses seguiram outros rumos, nomeadamente para a Física Nuclear [47, 48]. Mas Almeida Santos providenciou para que outro membro do corpo docente se fosse doutorar a Londres sob a supervisão de Kathleen Lonsdale e todo este trabalho pioneiro poderá estar na origem do actual Centro de raios X da Universidade de Coimbra [49]

\section{COMENTÁRIO FINAL}

Uma das autoras (AMVC) foi aluna do Professor Almeida Santos nos anos lectivos de 1962/63 e 63/64. Relembra um professor original, com sentido de humor, que fazia perguntas nas aulas a que só os mais afoitos tentavam responder. No início dos anos 1960, o uso da literatura científica não era muito incentivado na Universidade de Coimbra e os alunos também não estavam geralmente a par dos trabalhos de investigação dos seus professores. Após alguns anos que as autoras já levam de estudos com os aniões do tipo Keggin, não deixou de ser uma surpresa verificar a existência de participação portuguesa nos estudos estruturais iniciais sobre polioxometalatos, que desejamos partilhar. Só podemos especular se o anião de Keggin não poderia ter sido também o anião de Santos.

\section{AgRADECIMENTOS}

As autoras agradecem à Universidade de Aveiro e ao CICECO todas as facilidades concedidas para a realização deste trabalho.

\section{REFERÊNCIAS}

[1] M.T. Pope, "Heteropoly and Isopoly Oxometalates", Springer-Verlag, Berlin, 1983.

[2] M.T. Pope, A. Muller (eds.), "Polyoxometalates: from platonic solids to anti-retroviral activity”, Kluwer, Dordrecht, 1994

[3] M.T. Pope, A. Muller (eds.), "Polyoxometalate chemistry: from topology via self-assembly to applications”, Kluwer, New York, 2002

[4] J.J. Borrás-Almenar, E. Coronado, A. Muller, M.T. Pope (eds.), "Polyoxometalate Molecular Science”, Kluwer, Dordrecht, 2003

[5] C.L. Hill (ed.), Chem. Rev. 98 (1998) fascículo 1 (número temático)

[6] L. Cronin, A. Muller (eds.), Chem. Soc. Rev. 41 (2012) fascículo 22 (número temático)

[7] C.L. Hill (ed.), J. Mol. Catal. A: Chem. 262 (2007) fascículo 1-2 (número temático)

[8] A.M.V. Cavaleiro, in J.L. Figueiredo, M.M. Pereira, J. Faria (eds.), "Catalysis from theory to applications", Coimbra University Press, Coimbra, 2008, pp. 335-354

[9] T. Yamase, J. Mater. Chem. 15 (2005) 4773-4782

[10] Y. Song, R. Tsunashima, Chem. Soc. Rev. 41 (2012) 7384$-7402$

[11] D. Long, R. Tsunashima, L. Cronin, Angew. Chem. Int. Ed. 49 (2010) 1736-1758
[12] D.E. Katsoulis, Chem. Rev. 98 (1998) 359-387

[13] A.M.V. Cavaleiro, J.D. Pedrosa de Jesus, H.I.S. Nogueira, in P. Braunstein, L.A. Oro, P.R. Raithbay (Eds), "Metal Clusters in Chemistry”, vol. 1, VCH, Weinheim, 1999, pp. 444-458

[14] L.E. Briand, G.T. Baronetti , H.J. Thomas, Appl. Catal. A: General 256 (2003) 37-50

[15] H.N. Miras, J. Yan, D. Long, L. Cronin, Chem. Soc. Rev. 41 (2012) 7403-7430

[16] Y. Song, D. Long, C. Ritchie, L. Cronin, The Chemical Record 11 (2011) 158-171

[17] D. Fan, J. Hao, Q. Wei, J. Inorg. Organomet. Polym. 22 (2012) 301-306

[18] J.L.C. Sousa, I.C.M.S. Santos, M.M.Q. Simões, J.A.S. Cavaleiro, H.I.S. Nogueira, A.M.V. Cavaleiro, Catal. Commun. 12 (2011) 459-463

[19] F.M. Santos, P. Brandão, V. Félix, M.R.M. Domingues, J.S. Amaral, V.S. Amaral, H.I. S. Nogueira, A.M.V. Cavaleiro, Dalton Trans. 41 (2012) 12145-12155

[20] R.J.B. Pinto, C.M. Granadeiro, C.S.R. Freire, A.J.D. Silvestre, C.P. Neto, R.A.S. Ferreira, L.D. Carlos, A.M.V. Cavaleiro, T. Trindade, H.I.S. Nogueira, Eur. J. Inorg. Chem. (2013) 1890-1896

[21] J.F. Keggin, Nature 131 (1933) 908-909

[22] J.F. Keggin, Proc. R. Soc. Lond. A 144 (1934) 75-100

[23] M. Fournier, C. Feumi-Jantou, C. Rabia, G. Herve, S. Launay, J. Mater. Chem. 2 (1992) 971-978

[24] U.B. Mioc, M.R. Todorovic, M. Davidovic, P. Colomban, I. Holclajtner-Antunovic, Solid State Ionics 176 (2005) 3005$-3017$

[25] M. Misono, I. Ono, G. Koyano, A. Aoshima, Pure Appl. Chem. 72 (2000) 1305-1311

[26] M. Misono, Catal. Rev. Sci. Eng. 29 (1987) 269-321

[27] M. Misono, Chem. Commun. (2001) 1141-1152

[28] Trata-se da Victoria University of Manchester, conhecida por University of Manchester (1903-2004). A actual The University of Manchester resultou da fusão da Victoria University of Manchester com o University of Manchester Institute of Science and Technology (UMIST) em 2004.

[29] R.W. James in P.P. Ewald (Ed.), "Fifty Years of X-Ray Diffraction”, International Union of Crystallography, Utrecht, 1962, 420-429

[30] W.L. Bragg, Acta Cryst. A26 (1970) 173-177

[31] J.F. Keggin, Nature 132 (1933) 351

[32] G.M. Brown, M.-R. Noe-Spirlet, W.R. Busing, H.A. Levy, Acta Cryst. B33 (1977) 1038-1046

[33] J.W. Illingworth, J.F. Keggin, J. Chem. Soc. (1935) 575-580

[34] R. Signer, H. Gross, Helv. Chim. Acta 17 (1934) 1076-1080

[35] J.A. Santos, Proc. R. Soc. Lond. A 150 (1935) 309-322

[36] A.J. Bradley, J.W. Illingworth, Proc. R. Soc. Lond. A 157 (1936) 113-131

[37] J.F. Keggin, F.D. Miles, Nature 137 (1936) 577-578

[38] J.F. Keggin, G. Morris, A.M. Yuill, J. Text. Inst. 40 (1949) T702-T714 
[39] M.T. Pope, Eur. J. Inorg. Chem. (2013) 1561

[40] A.J.S. Fitas, E.V. Gomes, F. Nunes, J.P. Príncipe, comunicação apresentada na $4^{\text {th }}$ International Conference of the European Society for the History of Science, Barcelona, 1820 Novembro 2010

[41] B. Dawson, Acta Cryst. A6 (1953) 113-126

[42] H. Grime, J.A. Santos, Zeits. Krist. 88 (1934) 136-141

[43] J.W. Illingworth, J.A. Santos, Nature 134 (1934) 971

[44] J.A. Santos, J. West, J. Sci. Instrum. 10 (1933) 219-221

[45] V.Z. Sasca, O. Verdes, L. Avram, A. Popa, A. Erdohelyi, A. Oszko, Appl. Catal. A: General 451 (2013) 50-57

[46] M.H. Haider, N.F. Dummer, D. Zhang, P. Miedziak, T.E.
Davies, S.H. Taylor, D.J. Willock, D.W. Knight, D. Chadwick, G.J. Hutchings , J. Catal. 286 (2012) 206-213

[47] A.J.F. Leonardo, D.R. Martins, C. Fiolhais, Gazeta de Física 34/2 (1991) 9-15

[48] http://fisica.uc.pt/xt/pessoas/Homenagem_Almeida_Santos.pdf. Acedido em 15-04- 2013. Alternativamente consultar o site www.fis.uc.pt/ e seguir os links: pessoas $>$ emeritae $>$ homenagens.

[49] M.O. Figueiredo, M. Costa, A.M. Damas, M.T. Duarte, M.A. Carrondo, IUCR Newslett. 14/2 (2006) 8-13

[50] J.R. Santos, Revista da Faculdade de Ciências da UC 16 (1947) 5-66

[51] M.A. Parent, J. B. Moffat, Langmuir 12 (1996) 3733-3739

Actualidades Científicas

\section{SUBSTITUIR O INSUBSTITUIVEL}

O Bisfenol A (BFA) é um composto orgânico utilizado como monómero na produção de policarbonatos e de resinas epóxi, entre outros produtos. Os policarbonatos são utilizados na produção de materiais plásticos que contactam com os alimentos (por exemplo garrafas reutilizáveis, biberões e recipientes plásticos de armazenamento), enquanto as resinas epóxi são empregues no revestimento interno de latas e contentores de alimentos e bebidas. Portanto, o BFA presente nos recipientes de armazenamento pode ser transferido para os alimentos, contaminando-os, sendo posteriormente ingerido.

As análises que recorrem a testes de toxicidade padronizados têm provado até ao momento a segurança dos níveis actuais de exposição humana ao BFA, que são relativamente baixos. No entanto, os resultados de estudos toxicológicos recentes, que utilizam novas abordagens para testar os efeitos da toxicidade a longo prazo, levaram a que a Food and Drug Administration (FDA) tenha mostrado alguma preocupação sobre os possíveis efeitos do BFA no cérebro e na próstata de fetos, bebés e crianças. Por essa razão, a FDA, em cooperação com o Programa Nacional de Toxicologia americano, tem levado a cabo vários estudos aprofundados para responder a questões fundamentais e esclarecer dúvidas sobre os riscos do BFA para a saúde pública (disponível em http://www.fda.gov/newsevents/publichealthfocus/ ucm064437.htm).

Em Junho de 2011 a União Europeia proibiu o uso de BFA no fabrico de biberões e copos de plástico infantis e, em Outubro de 2012, a França tornou-se o primeiro país a alargar a proibição (em vigor a partir de 2015) do uso do BFA na produção de quaisquer materiais que estejam em contacto com os alimentos. É, por isso, imprescindível encontrar alternativas aos policarbonatos e resinas epóxi produzidos a partir do BFA. Não se trata, contudo, de uma tarefa simples. Por um lado, a substituição dos policarbonatos em biberões e copos infantis poderá ser uma tarefa relativamente fácil, uma vez que já existem no mercado poliésteres e polipropilenos que estão a ser utilizados em aplicações similares. No entanto, quanto a encontrar-se um produto alternativo às resinas epóxi - especialmente para o revestimento de latas - a questão torna-se mais complicada sob o ponto de vista técnico, constituindo, assim, um desafio para os próximos anos.

(adaptado do artigo BPA: friend or foe?, disponível em http://www.rsc.org/chemistryworld/2012/11/bpa-bisphenol)

Marcelo Vaz Osório (dcf20959@ff.up.pt)

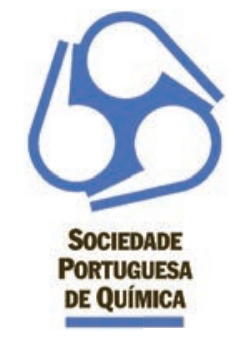

\section{SOCIEDADE PORTUGUESA DE}

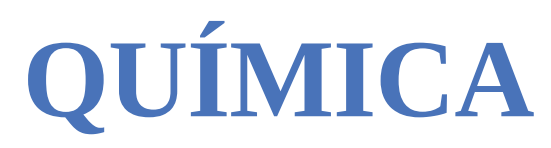




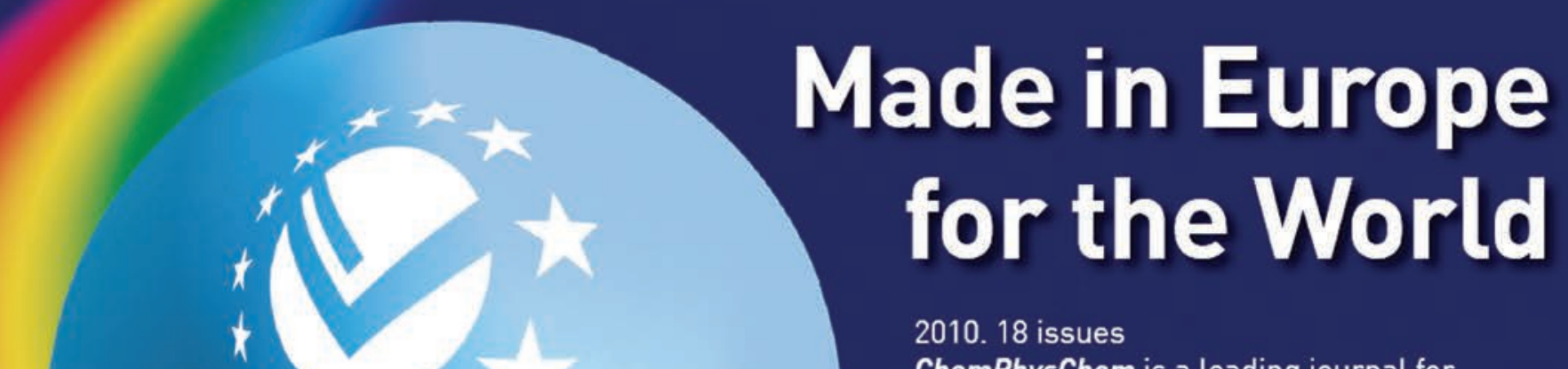

ChemPhysChem is a leading journal for chemical physics and physical chemistry. The journal offers an attractive mixture of Communications, Articles \& Minireviews www.chemphyschem.org

2010. 18 Issues.

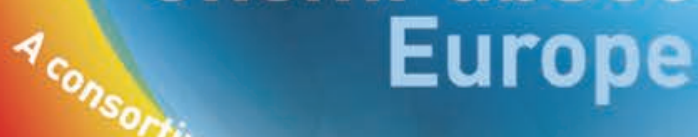

ChemBioChem is a leading journal for chemical biology and biological chemistry. The journal offers an attractive mixture of Communications, Full Papers, Reviews \& Minireviews www.chembiochem.org

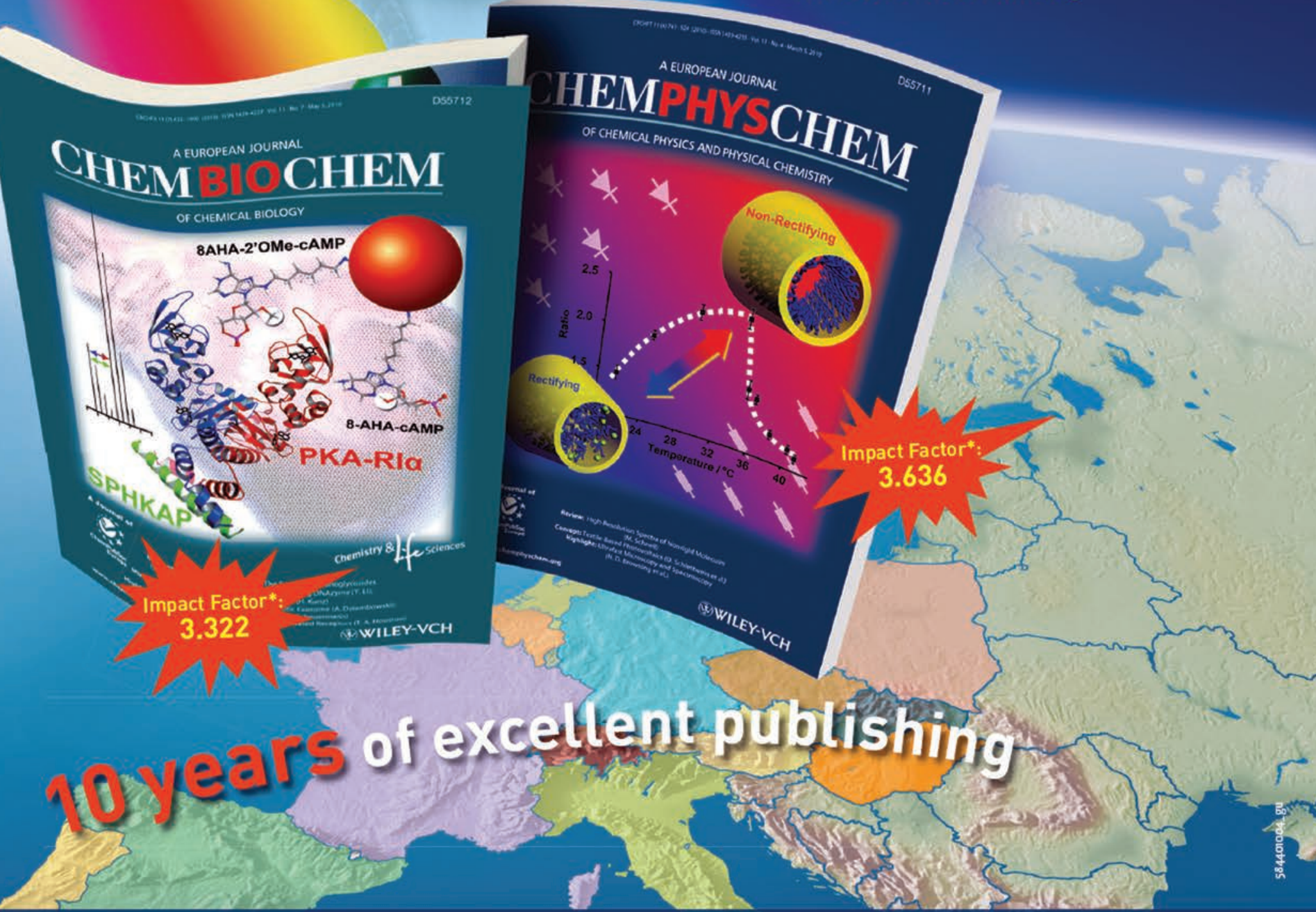

*2008 Journal Citation Reports ${ }^{\circledR}$ (Thomson Reuters, 2009]

\section{(WILEY-VCH}

For further information and to subscribe please send an e-mail to:

cs-journalsawiley.com

(Americas, Europe, Middle East and Africa, Asia Pacific)

servicelawiley-vch.de [Germany, Austria, Switzerland] cs-japandawiley.com (Japan) 